\title{
Comparative Study of Ultrasonography and Computed Tomography in Diagnosis of Acute Pancreatitis
}

\author{
Sneha Lalith ${ }^{1}$, Gurubharath Ilangovan ${ }^{2}$ \\ ${ }^{1}$ Junior Resident III, Department of Radiodiagnosis, ${ }^{2}$ Professor and HOD, Department of Radiodiagnosis, Shri Sathya Sai \\ Medical College and Research Institute, Ammapettai, Tamilnadu- 603108, India
}

Corresponding author: Dr. Sneha Lalith, No. 3, Garden Avenue, Officers Colony extn., Padi, Chennai-600050, India

DOI: http://dx.doi.org/10.21276/ijcmsr.2019.4.3.6

How to cite this article: Sneha Lalith, Gurubharath Ilangovan. Comparative study of ultrasonography and computed tomography in diagnosis of acute pancreatitis. International Journal of Contemporary Medicine Surgery and Radiology. 2019;4(3):C28-C33.

\section{A B S T R A C T}

Introduction: Pancreatitis is the inflammation of the Pancreas usually accompanied by abdominal pain and elevated levels of serum pancreatic enzymes with high rate of morbidity and mortality. USG provides initial radiological assessment, clue of the extent of involvement and an opportunity to evaluate other abdominal organs. CT scan provides a cross-sectional anatomy of the organ and involvement of adjacent structures. Study objective was to understand the role of CT and USG in determination of diagnosis of pancreatitis and to highlight and evaluate the cases in which USG failed to diagnose, which were helped through by CT.

Material and Methods: This study was done in the department of Radio diagnosis at SSSMCRI, Ammapettai, Tamilnadu, India, over a period of two years. USG of patients was done using Mindray with curvilinear transducer, high frequency linear array transducer and Doppler probe. CT scan was done using GE 16 Slice CT scan machine.

Results: Sensitivity of Ultrasonography in detecting acute pancreatitis was $88 \%$ in those patients in whom the pancreas was visualized. However, CT had a sensitivity of $100 \%$ visualization and better assessment of size. Though Ultrasonography is non-invasive, quick, inexpensive and a safe tool in diagnosis of pancreatic pathologies, it has certain limitations where pancreas may not be visualized. These limitations are overcome with the use of CT which yields more diagnostic information in the evaluation of both acute and chronic pancreatic pathologies.

Conclusion: Ultrasound is the first investigation of choice in a patient suspected to have acute pancreatitis. But ultrasonography has low sensitivity owing to poor penetration of ultrasound waves in obese patients and patients with excessive bowel gas. Early stages, extra pancreatic extension and complications may not be picked up with ultrasonography. CT has better sensitivity and specificity hence CECT forms a confirmative investigation in diagnosis and staging of pancreatic pathologies.

Keywords: Acute Pancreatitis, CT Scan, Modified CT Severity Index, Ultra Sonogram

\section{INTRODUCTION}

Pancreas is a dual gland in human body, difficult to evaluate, both by clinical and routine radiological methods. It is a retroperitoneal glandular organ in the upper abdomen, but in reality serves two purposes - an exocrine gland aiding in digestion and an endocrine gland producing hormones. Hence the evaluation of pancreas is complicated as well.

An inflammatory pathology involving the pancreas will form part of the differential diagnosis of other conditions presenting with acute abdominal pain. Earlier studies of conventional radiographs were non-specific for pancreatic pathologies. Imaging with ultrasound and computed tomography has afforded rapid, accurate and non-invasive evaluation of pancreas.

Ultrasonic devices for medical purposes were used in the year 1957. Ultrasonography provided the first reliable, reproducible, inexpensive, non-invasive, cross-sectional view of pancreatic anatomy and radiation free imaging, However, it has limitation in obese patients and in those with large amounts of bowel gas. ${ }^{1}$

Computed tomography invented in the year 1973, incorporates several unique features which facilitate pancreatic imaging in finer detail. ${ }^{2}$ Earliest investigators to evaluate the role of Computed Tomography in the assessment of pancreatic and Peripancreatic abnormalities were Haaga and Alfidi (1976). ${ }^{3}$ The improved spatial resolution and contrast sensitivity available with the present generation of CT scanners has further enhanced our capability for evaluating pancreatic lesions unlike angiography and ERCP which are difficult to perform as well as interpret. Although Ultrasound and Percutaneous transhepatic cholangiography (PTC) have a definite role in the evaluation of pancreatic lesions, Computed tomography with its higher sensitivity emerges as the imaging technique of choice for evaluating the nature and extent of pancreatic lesions.

Study objective was to understand the role of CT and USG in determination of diagnosis of pancreatitis and to highlight and evaluate the cases in which USG failed to diagnose, which were helped through by CT. 


\section{MATERIAL AND METHODS}

This study was a prospective study which was done in the department of Radiodiagnosis, Shri Sathya Sai medical college research institute, Ammapettai, Chennai in 50 patients over a period of 2 years (from 0ct 2016 - Oct 2018) after obtaining clearance from Institutional Ethical Committee. Informed written consent was obtained from the patients before undergoing USG and CT examination.

Ultrasound machine: Mindray DC7 with curvilinear transducer, high frequency linear array transducer and Doppler probe.

\section{Computed tomography: GE 16 SLICE}

\section{Methodology}

Study Population: This cross sectional study was done on patient which were referred to Department of Radiology, SSSMCRI, Ammapettai, with acute abdomen, supporting acute pancreatitis for CECT were first evaluated with Ultrasonography in our study period.

Study Period: Period of 2 years (from 0ct 2016 - Oct 2018) after obtaining clearance from Institutional Ethical Committee

Sampling Methods: Simple random selection as per inclusion and exclusion criteria

Inclusion Criteria: Patients of all age groups were referred for ultrasound abdomen in whom pancreatic pathology was detected on routine protocol.

Exclusion Criteria: Patients who were pregnant or expecting a pregnancy. Patient who were not willing to undergo the study. Other exclusion criteria for CT including. Previous history of hypersensitivity reaction. Bronchial Asthma. Impaired renal functions to undergo contrast examination

\section{Sample size}

$\mathrm{n}=\frac{\left(\mathrm{Z}^{2} \times \mathrm{p}(1-\mathrm{p})\right)}{\mathrm{e}^{2}}$

Where $Z$ = value from standard normal distribution corresponding to desired confidence level $(Z=1.96)$

$p$ is expected true population

$\mathrm{e}$ is desired population

We have included 50 cases in our study.

\section{Equipment}

The grey scale real time transabdominal ultrasound was performed using Mindray DC7 with a 3.5 MHz curvilinear transducer, high frequency linear array transducer and Doppler probe.

\section{USG scan technique for pancreas}

The TAS examination of the pancreas was performed on patients with overnight fasting, to improve the evaluation of the pancreas, patients were asked to drink $250-200 \mathrm{ml}$ of water prior to examination for which provided a sonic window into the pancreas. transverse and sagittal scan were performed.

\section{STATISTICAL ANALYSIS}

Data was collected, entered in MS excel sheet and was analyzed with standard statistical tests using SPSS software version 23.

\section{RESULTS}

\section{Age profile of patients with acute pancreatitis}

Study group included 50 patients selected from the patients sent for ultrasonography having probable acute pancreatitis. Patients of all age groups were imaged using USG, out of which 31 - 40 years of age patients peaked the charts (figure-1).

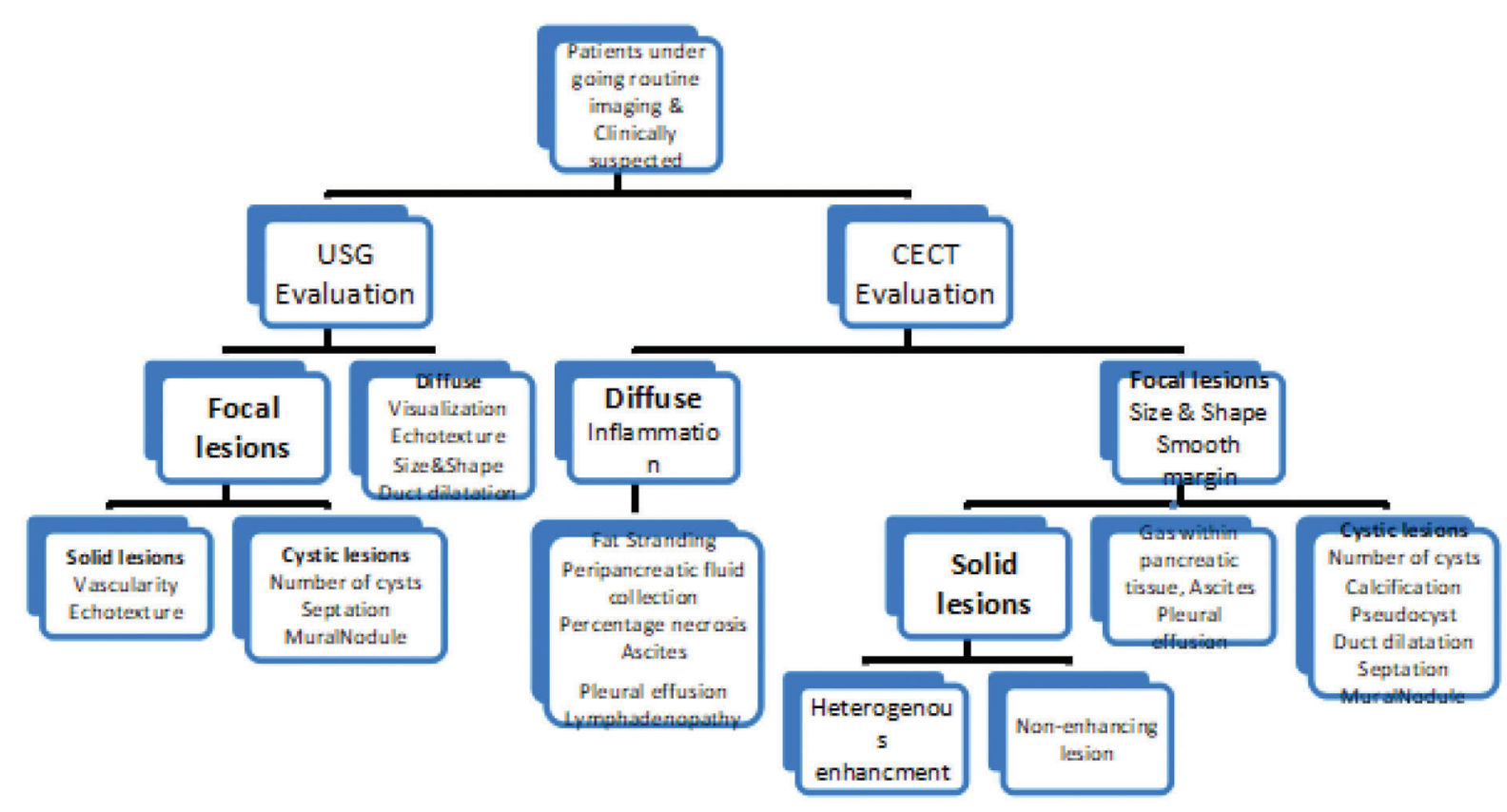

Table 1: Imaging in acute pancreatitis 


\begin{tabular}{|c|c|c|c|}
\hline \multicolumn{2}{|c|}{ Ultrasonography Appearance } & \multirow{2}{*}{$\begin{array}{c}\text { Number of cases } \\
44\end{array}$} & \multirow{2}{*}{$\begin{array}{c}\text { Percentage } \\
88 \%\end{array}$} \\
\hline Visualization & Fair to Excellent & & \\
\hline \multirow[t]{2}{*}{ Size } & Normal & 2 & $5 \%$ \\
\hline & Enlarged & 42 & $95 \%$ \\
\hline \multirow[t]{2}{*}{ Shape and Contour } & Normal & 44 & $100 \%$ \\
\hline & Altered & 0 & $0 \%$ \\
\hline \multirow[t]{3}{*}{ Echotexture } & Hyperechoic & 0 & $0 \%$ \\
\hline & Hypoechoic & 42 & $95 \%$ \\
\hline & Mixed & 0 & $0 \%$ \\
\hline Duct Dilatation & $\mathrm{MPD}>2.5 \mathrm{~mm}$ & 6 & $14 \%$ \\
\hline Gall stones & & 7 & $16 \%$ \\
\hline
\end{tabular}

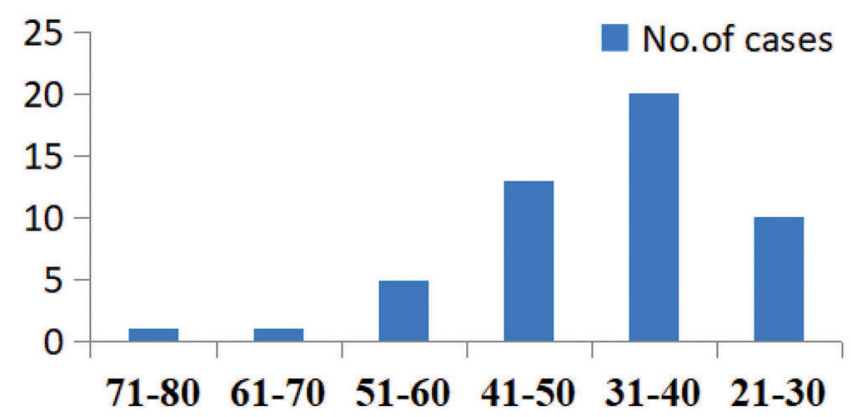

Figure-1: Age distribution

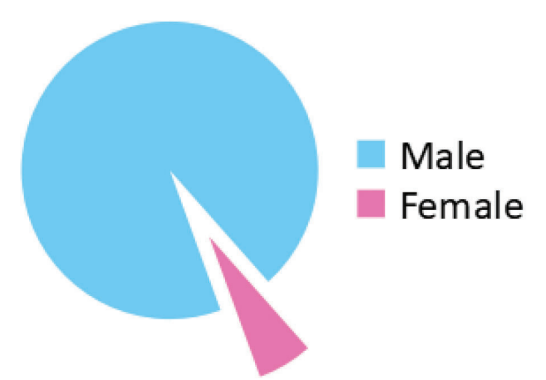

Figure-2: Sex distribution

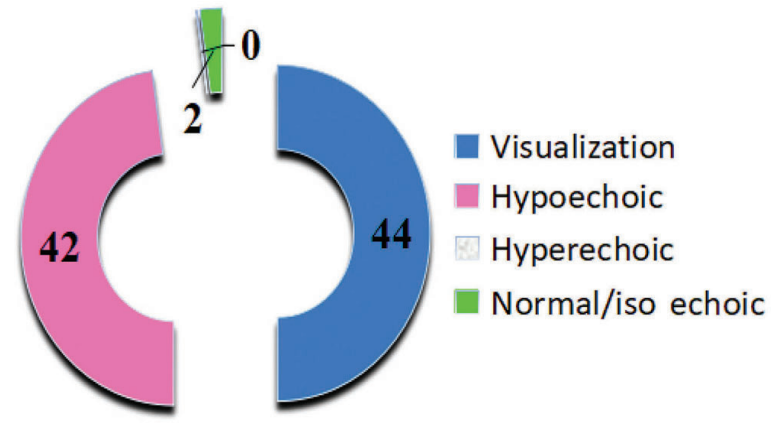

Figure-3: Based on visualization

\section{Sex distribution of pancreatic lesions}

Of the 50 patients selected in our study without sex distribution, male patients out numbered females totalling 94\% (figure-2).

\section{Based on visualization}

Among 44 of the 50 patients in whom pancreas was visualized, echotexture of each acute pancreatitis were individually tabulated. $95 \%$ of the cases which equals to 42 cases showed hypoechogenecity and 6 cases had duct dilatation (table-2).

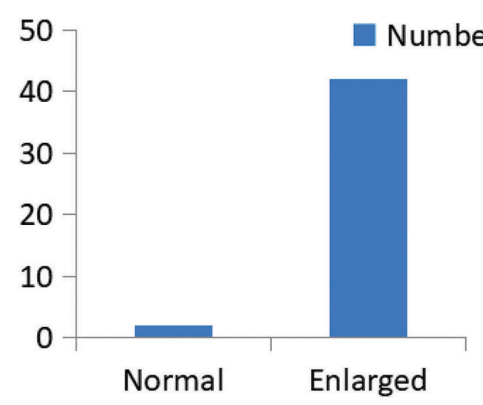

Figure-4: Based On Size

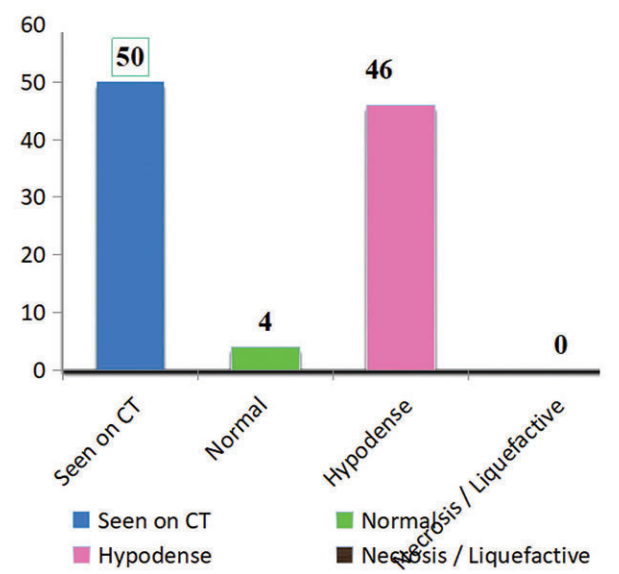

Figure-5: Based On Visualization

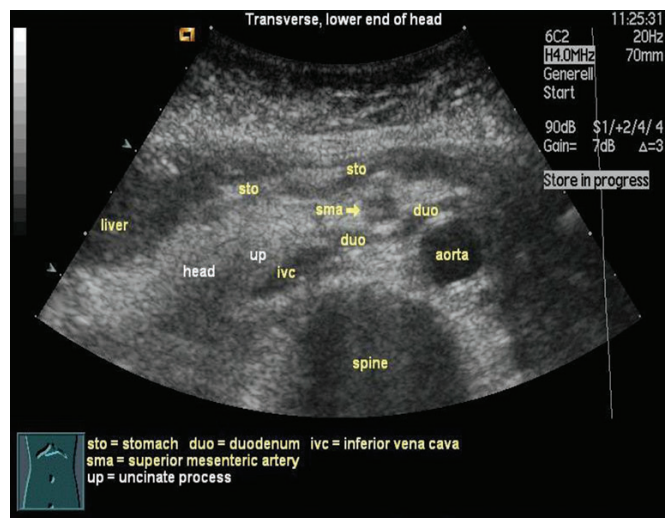

Figure-6: Normal USG image for visualization of pancreas

\section{Based on size}

Size of the pancreas was measured and assessed in 44 patients in whom pancreas was visualized in USG, out of which, 42 cases appeared enlarged and remaining 2 appeared normal in 


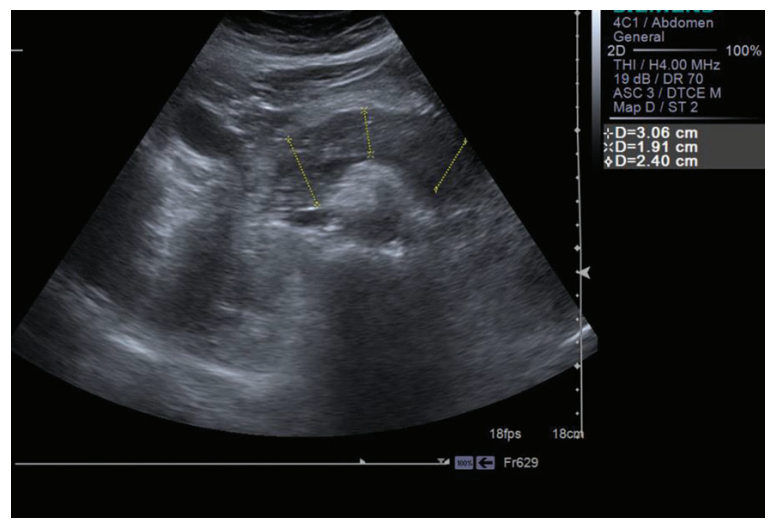

Figure-7: Bulky and Hypoechoic pancreas

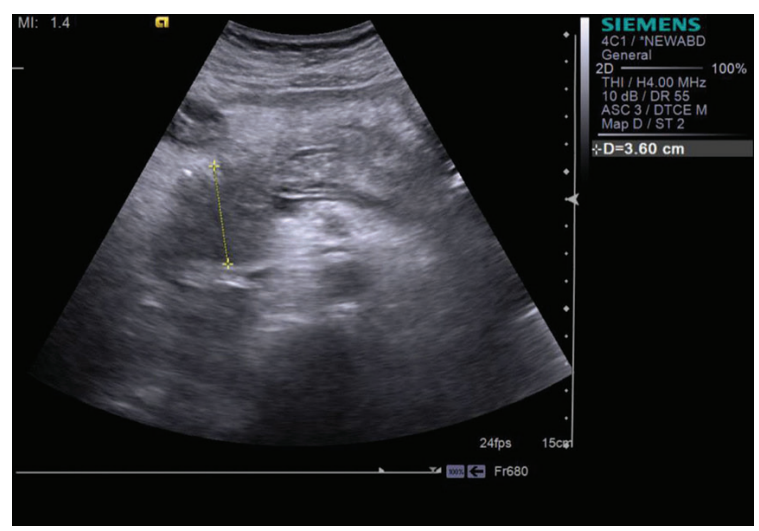

Figure-8: Bulky pancreas with thin rim of peripancreatic fluid and dilated MPD

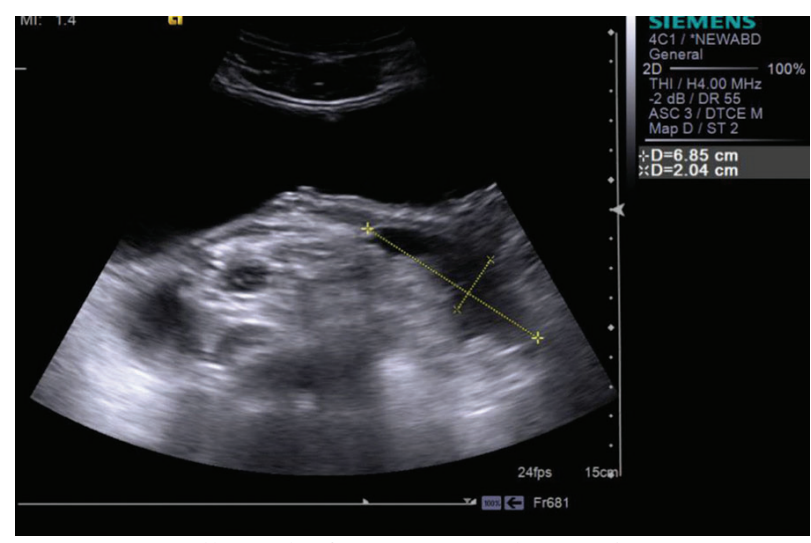

Figure-9: Bulky tail of pancreas with pseudo pancreatic cyst

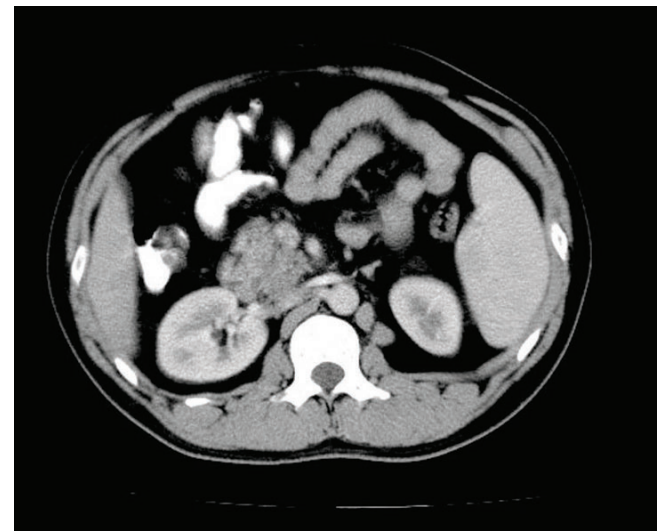

Figure-10: CECT Axial section showing oedematous uncinate process

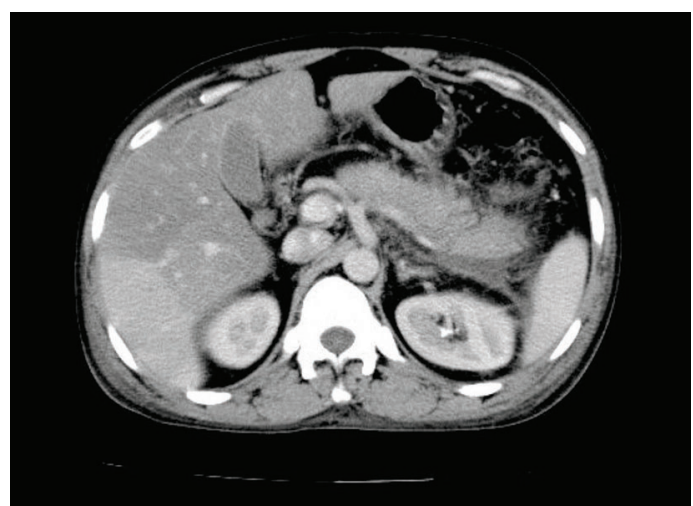

Figure-11: CECT Axial section showing body and tail of pancreas bulky with peripancreatic fat stranding.

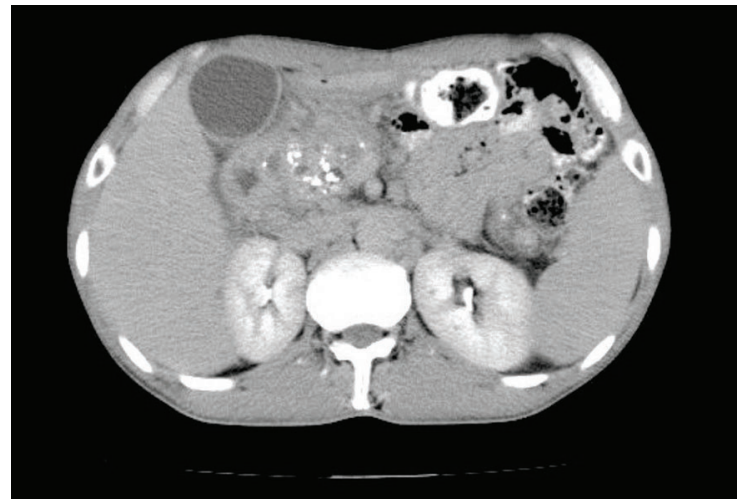

Figure-12: CECT Axial section showing bulky pancreas and intraparenchymal calcifications

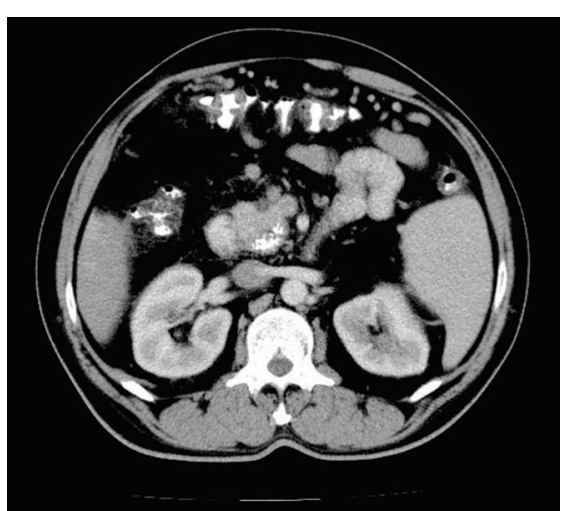

Figure-13: CECT Axial section showing enlarged pancreatic head with coarse calcifications

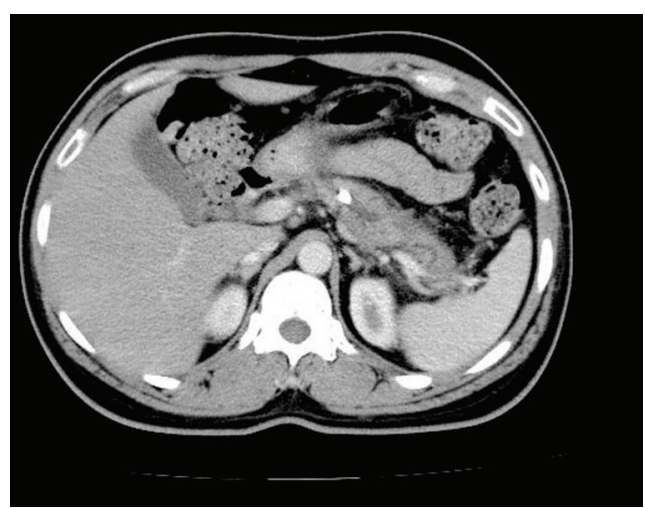

Figure-14: CECT Axial section showing dilation of main pancreatic duct with intra ductal calcification 
size (figure-3).

\section{Computed tomography appearance of acute pancreatits.}

Pancreas was visualized $100 \%$ in all the cases evaluated by computed tomography plain and contrast study. $92 \%$ of cases showed hypodensity in plain and contrast CT and 6 cases equalling $12 \%$ showed main pancreatic duct dilatation of more than $2.5 \mathrm{~mm}$ (figure-4).

\section{CT appearance of extra pancreatic findings}

Extra pancreatic findings including gall stones, calcification, ascites, pleural effusion, peripancreatic fluid collections and inflamed peripancreatic fat were all tabulated (figure-5).

\section{DISCUSSION}

Study was carried out in selected 50 patients who had various clinical presentations and was being evaluated for acute pancreatitis. Majority of patients with acute pancreatitis were between $31-40$ years of age (figure 1 ). The majority of patients with acute pancreatitis were males comprising 47 cases out of 50 cases representing $94 \%$ of the total.

Studies by Silverstein et $\mathrm{al}^{1}$ have noted that males with acute pancreatic pathologies were older (mean age 41 years) than females (mean age 32 years). In studies by Luetmer, Stephens and Ward et al and by Alpern et al have found that mean age of patients with chronic pancreatic pathologies were 54.3 years and 47 years respectively.

Alterations in size were better appreciated on CT. On CT, 48 patients with acute pancreatitis (96\%) were seen to have a bulky pancreas. Of the remainder, both the patients had normal size pancreas.

However, in all the patients, the ultrasound visualization was adequate and the observation of a dilated pancreatic duct and an atrophic pancreas was diagnostic of chronic pancreatic pathology. Hence, as suggested by L. Bolondi et al, ultrasound, should be first diagnostic step when pancreatic disease is suspected.

As mentioned in the study by SJ Hessel et al, a negative ultrasound study does not exclude significant and, at times, life-threatening pancreatic disease. ${ }^{9}$ Ultrasound may lead to a definite diagnosis and visualize complications of pancreatitis. In fact, the most accurate assessment of pancreatitis is achieved by a combination of clinical evaluation (symptoms and pancreatic function tests) and radiological definition of duct and parenchymal changes.

\section{Ultrasonography findings}

Pancreas was visualized by USG (figures 6-9) in 44 patients comprising $88 \%$ of the total which is far better than the previous studies by Calleja and J S Barkin ${ }^{2}$ which stated that in acute pancreatitis overlying bowel gas disturbance may obscure the pancreas in $40 \%$ of patients.

Enlarged pancreas is due to interstitial oedema within the pancreatic parenchyma. Bulky pancreas was seen in 42 patients (95\%) which is more than reported in R B Jeffery Jr, where only a third of patients with acute oedematous pancreatitis had enlarged gland. ${ }^{3}$ The presence of duct dilatation in pancreatic pathologies is very variable and could be compressed due to oedema or the hypoechoic pancreas may render the duct more easily visible., ${ }^{4,5}$ In this study, it was seen in almost 6 patients (14\%) of whom 2 cases were of acute on chronic pancreatitis. A bulky, hypoechoic pancreas is characteristic of oedematous pancreatitis. However, one series has shown this finding only in a third of patients with oedematous pancreatitis. ${ }^{6}$ In the present study, hypoechoic pancreas was seen in 42 patients (95\%).

\section{CT findings}

CT visualization (figures 10-14) of the pancreas was possible in all cases 50 patients (100\%) due to non-interference by the overlying bowel gas. CT can clearly give detailed information about the size of the pancreas without much hassle from gas or fat. ${ }^{7}$

This is the most reliable sign in pancreatic pathologies. The incidence of abnormal main pancreatic duct varies from $10 \%$ to $15 \%$ of cases. Our study showed this finding to be most common along with calcifications / calculi and was seen in 11 patients $(22 \%)$.

Comparison between usg and CECT in acute pancreatitis The overall visualization of the pancreas was far better by CT than by ultrasound. In a study done between 1979-1980 on 102 patients, good to excellent visualization of the pancreas was present in $64 \%$ of CT scans as compared to $20 \%$ of sonographic studies. ${ }^{8}$ With improvements in technology, visualization of the pancreas is better on CT modality. Our study shows that the pancreas is visualized in as many as $88 \%$ of patients on Ultrasonography and in $100 \%$ of patients on $\mathrm{CT}$ in acute pancreatitis.

Alterations in size were better appreciated on CT. On CT, 48 patients with acute pancreatitis $(96 \%)$ were seen to have a bulky pancreas. Of the remainder, both the patients had normal size pancreas.

Duct dilation and calcification were picked up in many patients on both modalities CT proved more useful in detecting free fluid as seen in 17 patients, in contrast to Ultrasound which picked up the same finding in 15 patients. Sensitivity of Ultrasonography in detecting acute pancreatitis was $88 \%$ in those patients in whom the pancreas was visualized. However, CT had a sensitivity of $100 \%$ visualization and better assessment of size. As all the patients had pancreatitis, the specificity could be estimated. Hence, as mentioned in the study by SJ Hessel et al, a negative ultrasound study does not exclude significant and, at times, life-threatening pancreatic disease. ${ }^{9}$

However, in all the patients, the ultrasound visualization was adequate and the observation of a dilated pancreatic duct and an atrophic pancreas was diagnostic of chronic

pancreatic pathology. Hence, as suggested by L. Bolondi et al, ultrasound, should be first diagnostic step when pancreatic disease is suspected. ${ }^{10}$

As mentioned in the study by SJ Hessel et al, a negative ultrasound study does not exclude significant and, at times, life-threatening pancreatic disease. ${ }^{9}$ Sensitivity was $100 \%$ for CT, higher than the sensitivity reported by L. Bolondi et al which was $70 \%$.

Ultrasound may lead to a definite diagnosis and visualize complications of chronic pancreatitis. In fact, the most accurate assessment of chronic pancreatitis is achieved by a combination of clinical evaluation (symptoms and pancreatic function tests) and radiologic definition of duct 
and parenchyma changes.

\section{Limitations}

It has certain limitations due to bowel gas where pancreas may not be visualized. These limitations are overcome with the use of CT which yields more diagnostic information in the evaluation of both acute and chronic pancreatic pathologies.

Intraobservership variation and limited sample size are the limitations of our study.

\section{CONCLUSION}

Diagnosis of a specific pancreatic pathology is often delayed because clinical clues from history and presentation of abdominal pain are ascribed to all the conditions of acute abdomen in the primary care setting. Also certain blood investigations like serum amylase and serum lipase though very specific for pancreatic pathologies can be normal in early stages of certain individuals. Similarly, Ultrasonography has certain limitations causing poor visibility which can be misleading even though it is less expensive, non-invasive and contain no harmful radiation.

CECT Abdomen, therefore, is able to detect most of the findings in relation to pancreatic pathologies. Ultimately all the patients with clinical suspicion of pancreatic pathology should benefit from CECT of Abdomen. Contrast enhanced CT scans are essential for the diagnostic work up since certain pathologies are classified based on parenchymal status and confident diagnosis can often be arrived by CECT alone or in correlation with clinical examination.

Ultrasonography visualized pancreas on about $88 \%$ patients whereas CT visualized pancreas in 100\% patients. Ultrasonography is non-invasive, quick, inexpensive widely available and a safe tool having no harmful radiation in the imaging and diagnosis of pancreatic pathologies. Extra pancreatic spread of inflammation and vascular complications may not be easily picked up by Ultrasonography due to its limitations. CECT forms a confirmative investigation in diagnosis and staging of Acute or Chronic pancreatic pathologies. Alteration in the size and echogenicity were the most common Ultrasonography findings.

Bulky hypoechoic pancreas was considered diagnostic of acute pancreatic pathologies on ultrasonography. Thus it is seen that both Ultrasonography and CT have roles to play in the diagnosis of pancreatitis and both are complementary to each other.

\section{REFERENCES}

1. Silverstein W., M. B. Isikoff, M. C. Hill, J. Barkin. Diagnostic Imaging of Acute Pancreatitis Prospective Study Using CT and Sonography. AJR 1981; 137(3): 497-502.

2. Calleja G.A., J.S Barkin. Acute Pancreatitis Medical Clin North Am 1993; 77 (5): 1037-1055.

3. Jeffrey RB Jr. Sonography in Acute Pancreatitis. Radiol Clin N Am 1989; 27 (1): 5- 17.

4. Hessel S.J. et al. A Prospective Evaluation of Computed Tomography and Ultrasound of the Pancreas. Radiology 1982; 143(5): 129-133.

5. Calleja G.A., J.S Barkia. Acute Pancreatitis. Medical
Clin North Am 1993; 77 (5): 1037-1055.

6. Jeffrey RB Jr. Sonography in Acute Pancreatitis. Radiol Clin N Am 1989; 27(1):5- 17.

7. Charles J. Yeo, John L Cameron. Exocrine Pancreas in Sabiston Text book of Surgery 17th Edition. Saunders Company 2005, 1643 - 1678.

8. Luetmer P.H., D.H. Stephens, E.M Ward. Chronic pancreatitis reassessment with current CT. Radiology 1989; 171(4): 353-357.

9. Hessel S.J. et al. A Prospective Evaluation of Computed Tomography and Ultrasound of the Pancreas. Radiology 1982; 143(1): 129-133.

10. Balthazar E.J. Complication of acute pancreatitis clinical and CT evaluation. Radiol Clin North Am 2002; 40(5): 1211-1227.

Source of Support: Nil; Conflict of Interest: None

Submitted: 18-03-2019; Accepted: 23-06-2019; Published online: 19-07-2019 